\title{
Neutron Computed Tomography of Plasma Facing Components for Fusion Experiments
}

B. Schillinger ${ }^{a,{ }^{*}}$ H. Greuner ${ }^{b}$, Ch. Linsmeier ${ }^{b}$

a Technische Universität München, FRM II and Faculty for Physics E21, Lichtenbergstr.1, 85748 Garching, Germany

${ }^{b}$ Max-Planck-Institut für Plasmaphysik, EURATOM Association, Boltzmannstr. 2, 85748

Garching , Germany

*Corresponding author: Dr. Burkhard Schillinger

Burkhard.Schillinger@frm2.tum.de

Lichtenbergstrasse 1, D-85748 Garching, Germany.

Tel: +49 89289 12185; Fax: +49 8928914997

Abstract: In nuclear fusion experiments, divertor plates are used to remove energy and particles from the plasma. These divertor plates can be made of water-cooled copper heat sinks covered by carbon fiber composite (CFC) protection tiles. During operation, surface temperatures in excess of $1000^{\circ} \mathrm{C}$ are reached for typical heat loads of $10 \mathrm{MW} / \mathrm{m}^{2}$. The large mismatch in the coefficients of thermal expansion for CFC and Cu causes high stresses and possibly bonding defects. Growing joint defects, which lead to unacceptable overheating of the protection tiles, are critical for the lifetime of the components.

A prototype component was subjected to 10.000 cycles at $10 \mathrm{MW} / \mathrm{m}^{2}$ to study the crack grow mechanism. Neutron computed tomography offers up the possibility to analyse such structures on centimetre-sized samples non-destructively with a high spatial resolution. At the ANTARES neutron imaging facility of the FRM II reactor, the samples were loaded with a contrast agent and examined with neutron computed tomography.

Keywords: Neutron Computed Tomography, Plasma Facing Components, Fusion, Divertor, Delamination. 


\section{Introduction}

Fusion energy is a promising energy source for the future. In the fusion experiment Wendelstein 7-X (W7-X) [1], which is under construction in Greifswald (Germany), and also in the future reactor experiment ITER [2], water-cooled components are employed in the socalled divertor to remove energy and particles from the plasma. The W7-X components are based on a water-cooled heat sink structure, which is protected by ceramic-like CFC tiles as plasma facing material (Fig.1). To bond these two materials a patented Cu casting technology was developed by Plansee SE (Austria) $[3,4]$.

Fig. 1. Design of the W7-X divertor target. A water-cooled copper base is protected by carbon fiber composite tiles.

During operation in a hydrogen plasma at heat flux densities of up to $10 \mathrm{MW} / \mathrm{m}^{2}$ the CFC tiles reach surface temperatures in excess of $1000^{\circ} \mathrm{C}$. The large mismatch in the coefficients of thermal expansion for CFC and $\mathrm{Cu}$ causes high stresses and possibly bonding defects. Growing joint defects, which lead to unacceptable overheating of the protection tiles, are critical for the lifetime of the components.

For an experimental investigation of the crack growth mechanism cyclic loading tests were performed with heat loads similar to the expected operational load. The investigated sample was loaded with 5.000 cycles at $10 \mathrm{MW} / \mathrm{m}^{2}, 10 \mathrm{~s}$ duration in the high heat flux test facility GLADIS at the IPP Garching (Fig.2). A detailed description of the facility is given in the reference [Ref. GLADIS].

Fig. 2. Heat load tests performed at GLADIS facility. The right image shows the sample during $10 \mathrm{MW} / \mathrm{m}^{2}$ heat loading. The neutral beam dump is located in the background.

Extensive metallographic and micro-chemical post-examinations were performed to analyse changes of the CFC structure and of the CFC/Cu interface. All these analyses are based on two-dimensional investigations only. Therefore they do not yield sufficient insight into the spatial distribution of structural material modifications and of joint failures. [6]

Fig. 3 shows local overheating during heat loading. A microscopical cut of the material shows clear delamination. Due to the 2D information in the inspection of the cut materials by optical microscopy, both the real extension of delamination and the development of cracks in the bulk CFC material cannot be assessed.

Fig. 3. IR image of the CFC tiles of the sample during heat loading. The position of the tomography sample is marked. Local overheating results from delamination between CFC tile and cooling structure. 


\section{Experimental}

\subsection{Preparation of samples}

Contrary to X-rays, neutrons can penetrate most metals up to a few $\mathrm{cm}$ thickness while giving a lot of contrast on many light elements. In addition, the element gadolinium has an extremely high absorption coefficient for neutrons, which may be used in a liquid contrast agent. For examination at the ANTARES neutron imaging facility [7] of the FRM II reactor of Technische Universität München, two cutout slices from divertor plates with about $5 \mathrm{~mm}$ width, $50 \mathrm{~mm}$ length and $7 \mathrm{~mm}$ thickness were prepared, one from an unloaded part of a test sample, one from an extensively heat loaded part Both samples were immersed in a test tube with liquid gadolinium contrast agent and placed into a vacuum chamber as a whole. The pressure was decreased to the point just before boiling of the liquid, so air contained in the cracks and in the hollow spaces of the CFC structure expanded and left the samples. Re-pressurizing of the chamber then forced liquid into the vacated hollow spaces of the immersed samples. The samples were then rinsed in water to remove contrast agent from the surface.

\subsection{Neutron Computed Tomography}

The samples were mounted on a rotation table at the ANTARES facility. The employed beam has a collimation of $L / D=800$, and a neutron flux of $2.6 * 10^{7} \mathrm{n} / \mathrm{cm}^{2} \mathrm{~s}$. 800 projections were recorded with a cooled CCD camera and neutron scintillation screen, computed tomography was calculated by filtered backprojection with a parallel beam algorithm. Fig.4 shows two tomographic slices of the samples at different heights. The heat loaded sample is at the lower left, the untreated sample at the upper right. With the selected parameters, the carbon fibers deliver rather low contrast and are nearly invisible, the copper is visible as a faint grey, but the contrast agent appears as strong white. Strands of many carbon fibers form an interwoven matrix with hollow spaces which are filled out by the contrast agent. Individual fibers are too small to be visible at the given spatial and contrast resolution. At the heat loaded sample, a large delamination area is filled with contrast agent, also revealing the conical laser holes. The unloaded sample shows no delamination at all.

Fig. 4. Two tomographic slices of the heat loaded and unloaded sample. The contrast agent fills the hollow spaces in the carbon fiber matrix and in the delamination area.

Fig. 5 and Fig. 6 show three-dimensional views of the computed tomography. The orientation is sideways compared to Fig. 4 , the slices of Fig. 4 are perpendicular to the image plane of Fig. 5 and 6, cutting the samples as cross section. The structure of the CFC matrix is revealed by its hollow spaces filled with the contrast agent, and delaminated regions are visible on the upper left. 
Fig. 5. Three-dimensional sideway view of the samples. The bright parts represent the hollow spaces filled with contrast agent.

Fig. 6. A different three-dimensional sideway view. Delamination is visible on the upper left.

\section{Evaluation and outlook}

The experiment demonstrates the possibility to analyse structures on centimetre-sized samples non-destructively with a high spatial resolution by neutron tomography. The obtained results confirm that the delamination occurs in the CFC / Cu interlayer during heat loading. The size of delamination is acceptable after such high number of high heat flux pulses. As a main result, a comparison of the CFC structures of the loaded and unloaded samples shows no difference or deterioration of the loaded sample. The integrity of both the bonding interface and the CFC structure could be confirmed even after extensive heat loads up to the expected lifetime of the components.

New samples of a new prototype loaded with 10,000 heat cycles have already been prepared, and the vacuum infiltration setup has been improved. Fine tuning of the pressure proved necessary to extract as much air as possible from the voids; the process took up to a quarter of an hour before no more gas bubbles were visible.

New tomographic examinations are under way; a quantitative evaluation will require additional samples, and additional work by the material scientists.

Neutron computed tomography has so far been the only tool to examine the 3D problem at hand, and aid the improvement of the manufacturing process.. Further examinations may follow for Wendelstein 7-X as well as the future ITER experiment.

\section{References}

[1] Renner, et al.: The capabilities of steady state operation at the stellarator W7-X with emphasis on divertor design. Nuclear Fusion, Vol.40 (2000) 1083-1093

[2] www.iter.org

[3] Patent EP 0663670. 257

[4] Patent TO2003A000795.

[5] H.Greuner et al., Design, performance and construction of a $2 \mathrm{MW}$ ion beam test facility for plasma facing components, Fusion Eng. Des. 75-79 (2005) 345-350.

[6] Greuner, H., B. Böswirth, J. Boscary, T. Friedrich, C. Lavergne, Ch. Linsmeier, J. Schlosser and A. Wiltner: Review of the high heat flux testing as an integrated part of W7-X divertor development. In: Fusion Engineering and Design 84, 2-6, 848-852 (2009) [7] B. Schillinger, E. Calzada, K. Lorenz, "Modern neutron imaging: Radiography, Tomography, dynamic and phase contrast imaging with neutrons", Solid State Phenomena Vol. 112 (May 2006) pp.61-72, Transtech Publications, online at www.scientific.net 


\section{CAPTION LIST:}

\section{Design of divertor target}

\section{Target element}
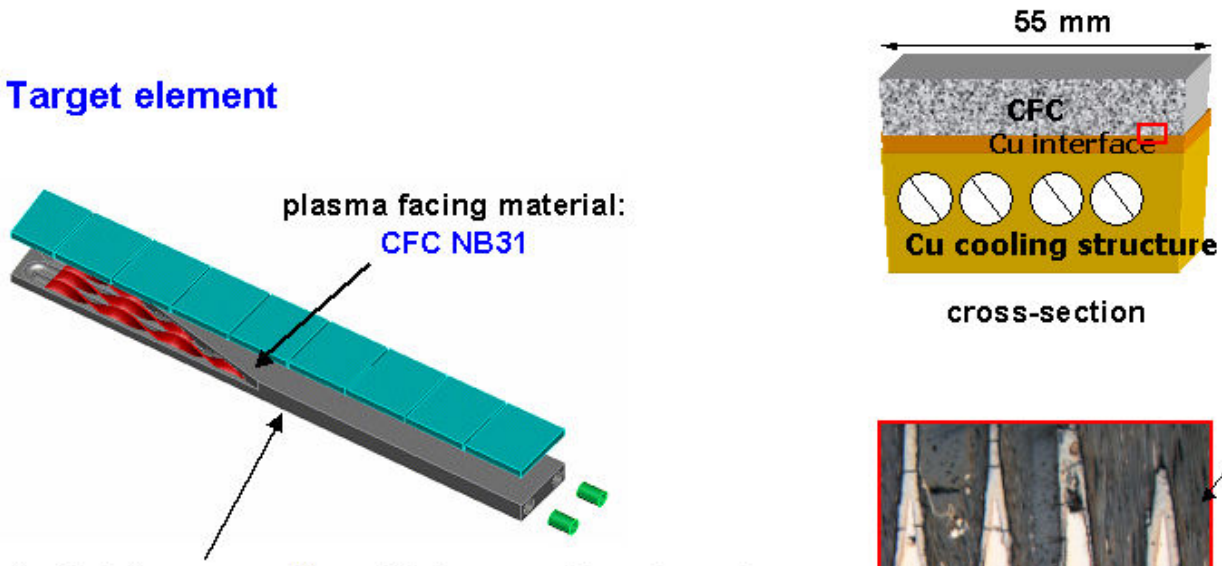

heat sink: copper alloy with inner cooling channel

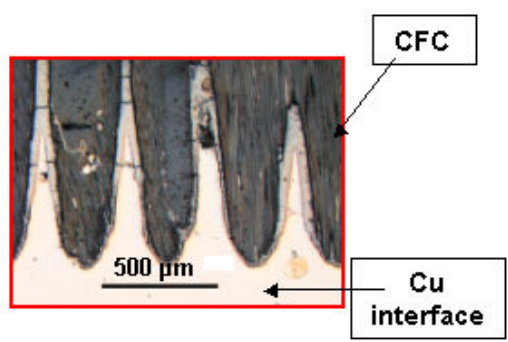

Magnification of $\mathrm{CFCl} \mathrm{Cu}$ interface

Fig. 1. Design of the W7-X divertor target. A water-cooled copper base is protected by carbon fiber composite tiles.
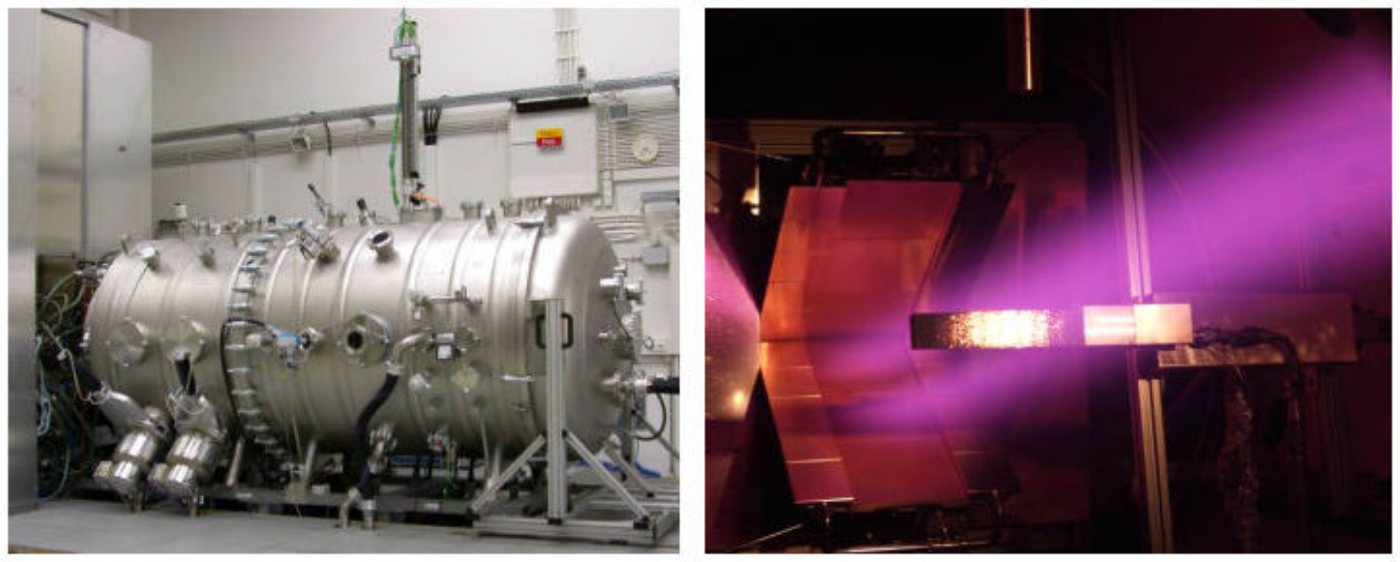

Fig. 2. Heat load tests performed at GLADIS facility. The right image shows the sample during $10 \mathrm{MW} / \mathrm{m}^{2}$ heat loading. The neutral beam dump is located in the background. 


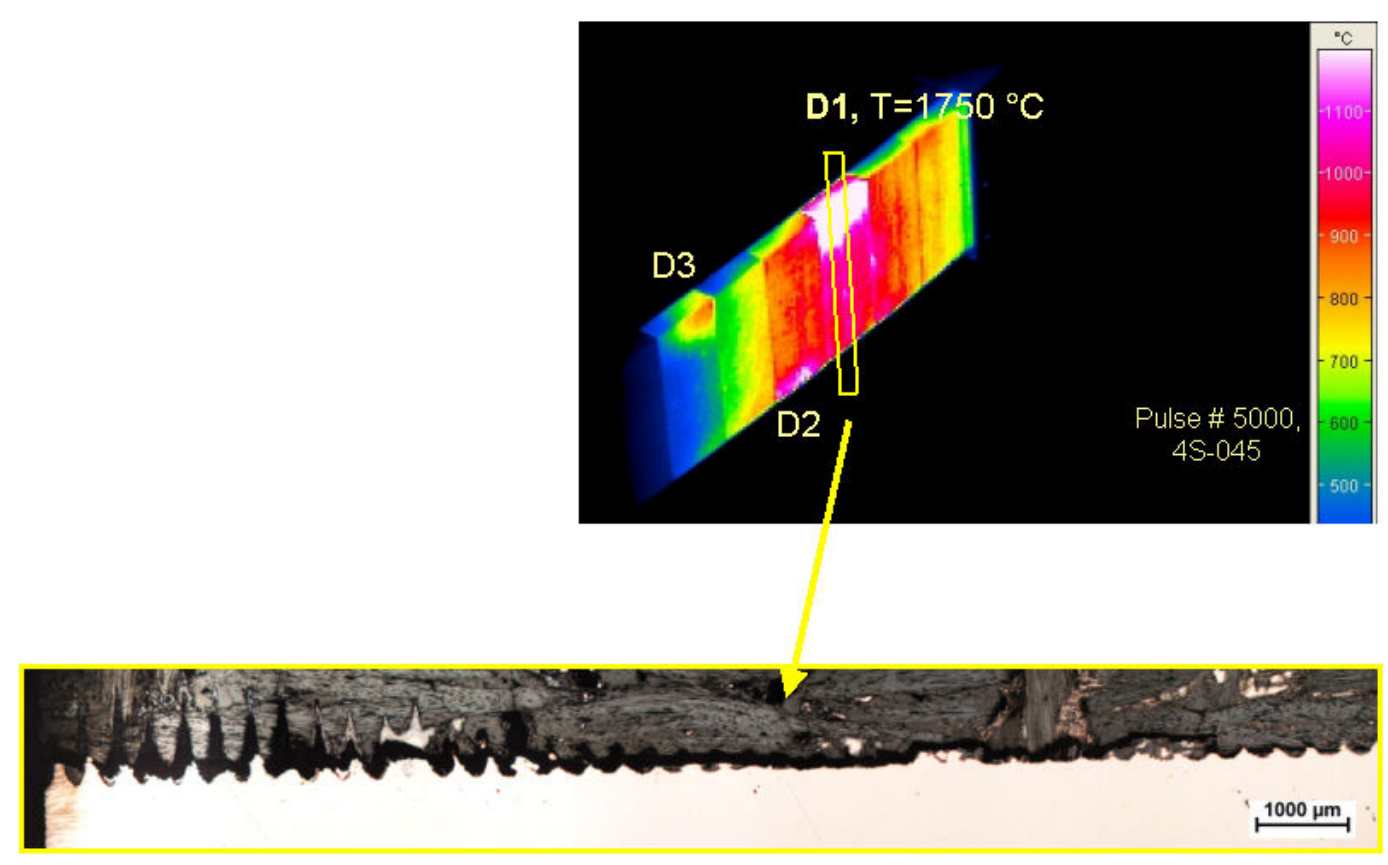

Fig. 3. IR image of the CFC tiles of the sample during heat loading. The position of the tomography sample is marked. Local overheating results from delamination between CFC tile and cooling structure.
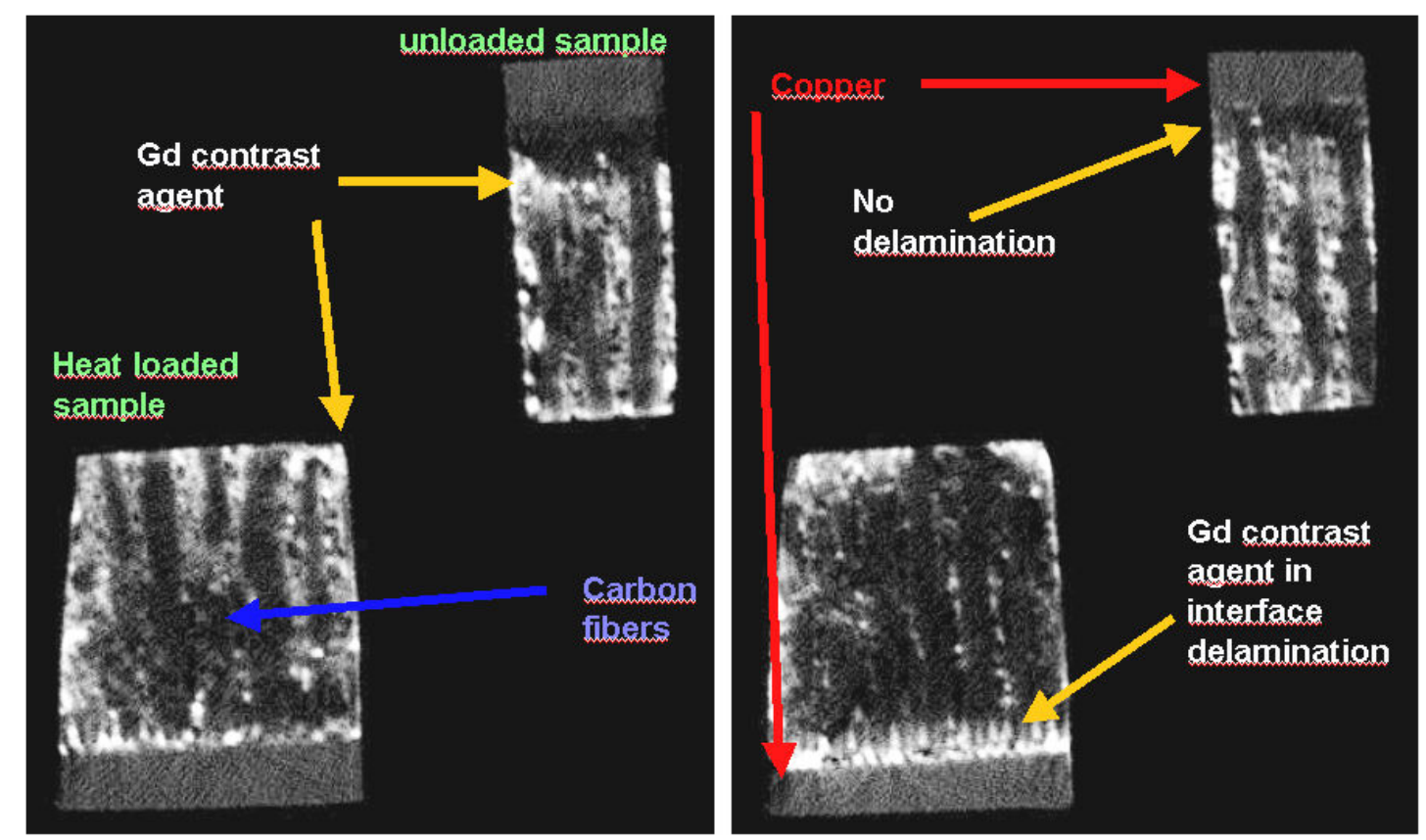

Fig. 4. Two tomographic slices of the heat loaded and unloaded sample. The contrast agent fills the hollow spaces in the carbon fiber matrix and in the delamination area. 


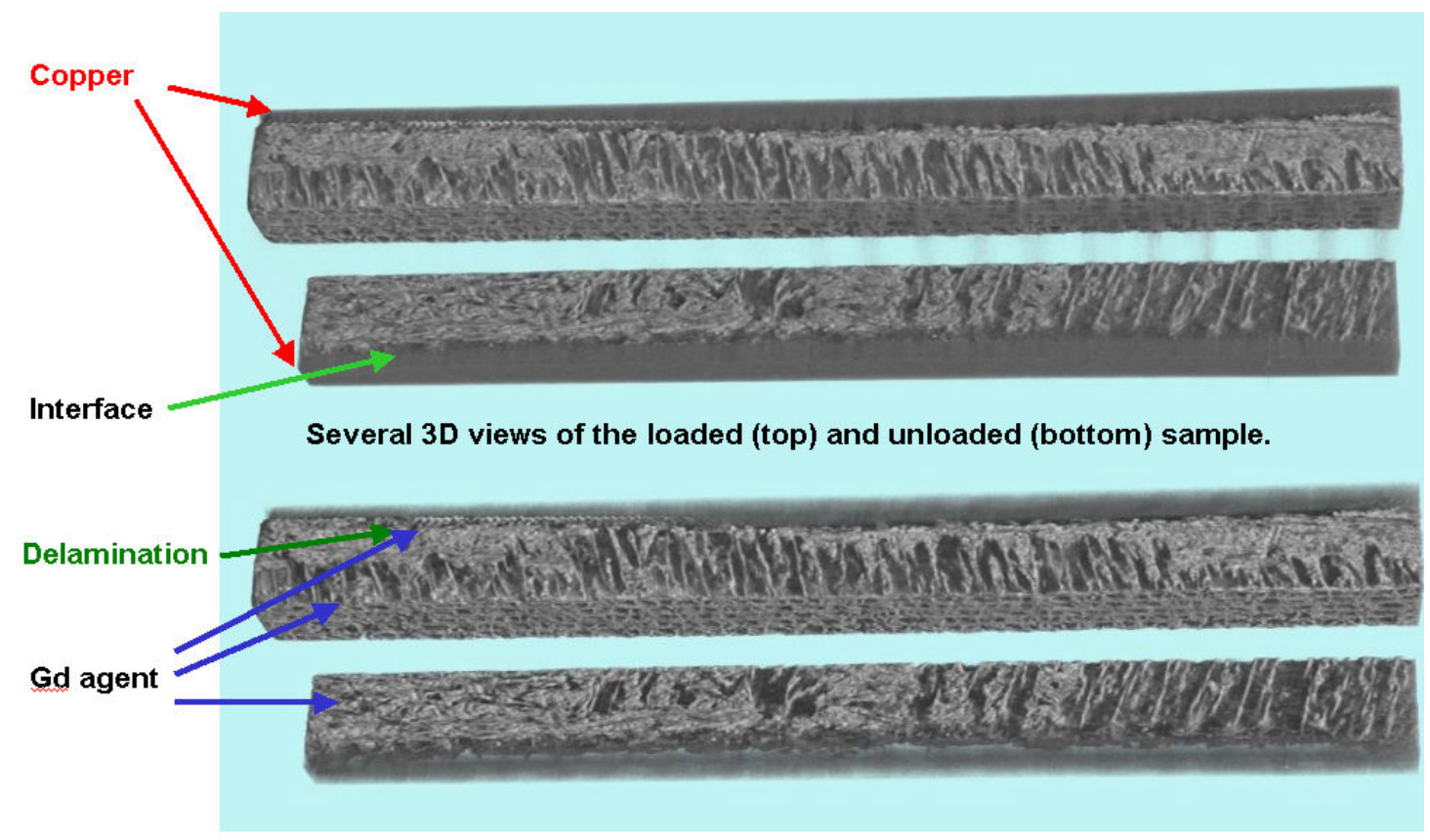

Fig. 5. Three-dimensional sideway view of the samples. The bright parts represent the hollow spaces filled with contrast agent.

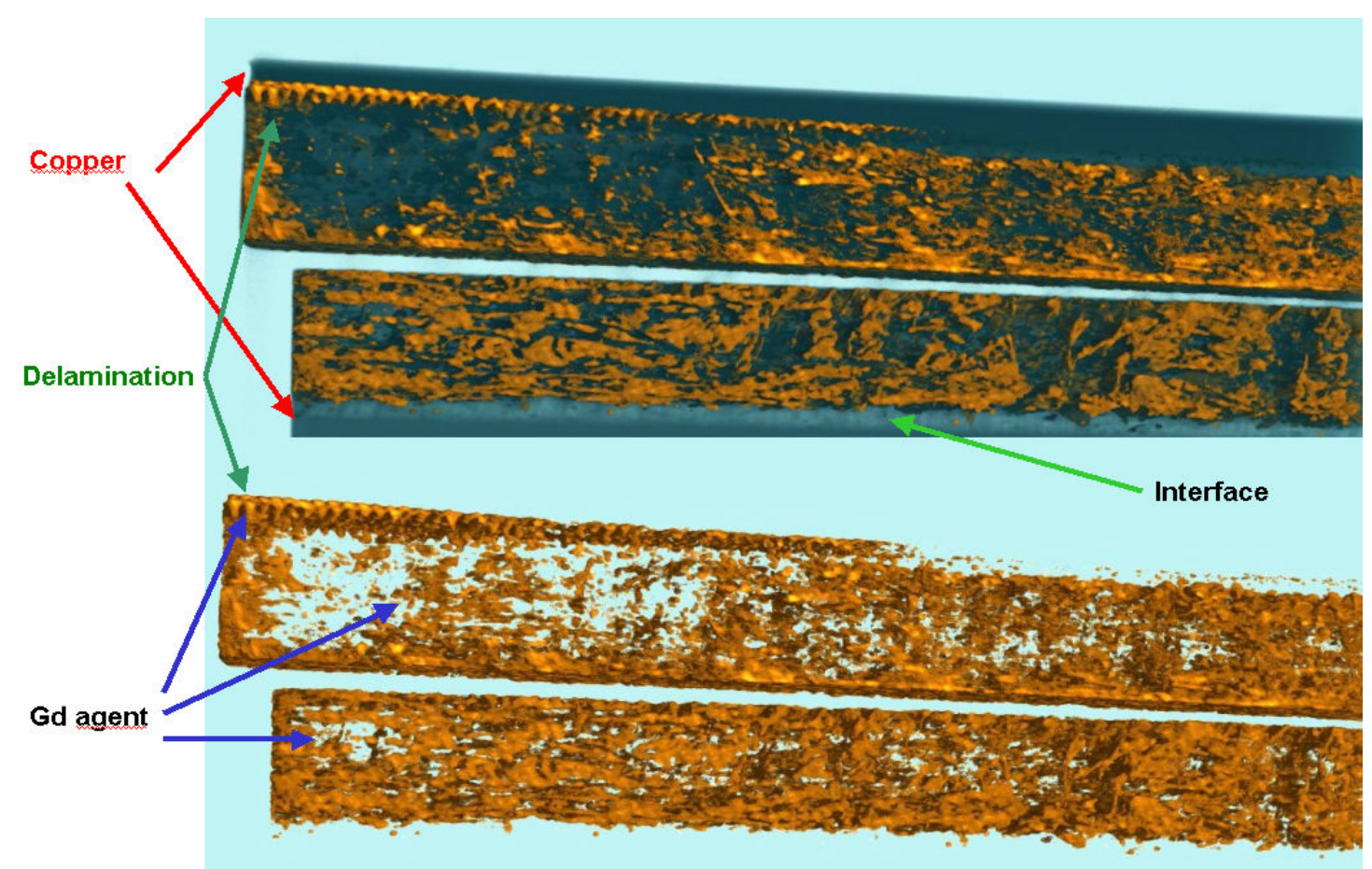

Fig. 6. A different three-dimensional sideway view. Delamination is visible on the upper left. 